\title{
Kein Anhängsel von \\ Wirtschaft und Verwaltung
}

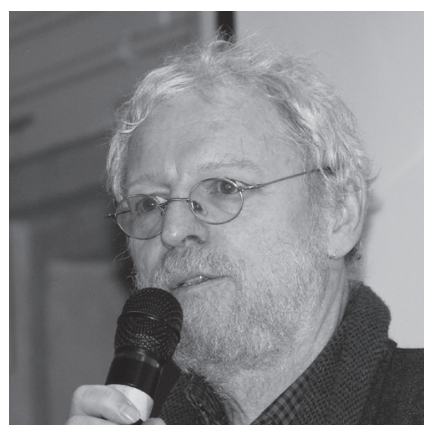

VON ARMIN WÖHRLE

Prof. Dr. Armin Wöhrle lehrt Sozial-

management an der Hochschule Mittweida.

E-Mailwoehrle@hs-mittweida.de

\author{
Die Entwicklung der Sozialwirtschaft zu einem \\ bedeutenden gesellschaftlichen und wirtschaftlichen \\ Sektor zeigt sich auch im Bemühen, theoretische \\ Konzepte für das Management sozialer Dienste und \\ Einrichtungen zu formulieren. Eine vorläufige Bilanz \\ kann zeigen, dass diese Versuche durchaus hilfreiche \\ Anregungen für die Praxis der Unternehmensführung \\ in der Sozialwirtschaft zeitigen können.
}

In Gesprächen mit dem Leitungspersonal in der Sozialwirtschaft wird oft zugegeben, dass eine Vielfalt an Managementinstrumenten und Managementtools in den organisationalen Gliederungen Einzug gehalten hat. Jedoch wisse niemand genau zu sagen, ob daraus bereits ein Managementkonzept entstanden sei.

Es wurde externe Beratung eingebunden und Organisationsentwicklungsprozesse vollzogen. Qualitätsmanagement wurde eingeführt, Personalentwicklungskonzepte wurden unverzichtbar, es gibt ein Leitbild, unter Umständen wurden Zielvereinbarungen geschlossen, über das Controlling hinaus wird mit einer Balanced Scorecard gearbeitet usw. Doch zeigen all die eingeführten Instrumente in die gleiche Richtung oder sind gar welche darunter, die sich gegenseitig verdoppeln und den organisatorischen Aufwand nur erhöhen oder arbeiten manche sogar gegeneinander, verlangen Ergebnisse, die nicht deckungsgleich zu bekommen sind?

Wer kann hier für seine Organisation systematisch begründete Antworten geben? Wer es kann, müsste von einem Managementkonzept in der Sozialwirtschaft sprechen können. Veröffentlichungen über theoretische begründete, stringente Managementkonzepte in der Sozialwirtschaft sind kaum zu finden.
Die Lehrenden in den über einhundert Studiengängen des Sozialmanagements und der Sozialwirtschaft (Boeßenecker/ Markert 2011) sind gegenwärtig über die Internationale Arbeitsgemeinschaft Sozialmanagement/Sozialwirtschaft (INAS) dabei, Übersichten zu den Studiengängen, zur Forschung und Entwicklung sowie Theoriebildung herzustellen. Dies geschieht durch ein Lexikon der Sozialwirtschaft (Maelicke 2008), auf Kongressen und in entsprechenden Veröffentlichungen. Auch erste Bilanzen werden dabei gezogen (Bassarak/ Wöhrle 2008, Grunwald 2009).

Ein besonderes Interesse ist dabei den Sozialmanagementkonzepten gewidmet. Nach einem Call-for-paper zum Thema Managementkonzepte für die Sozialwirtschaft, das an alle Fakultäten gerichtet war, die für das Sozialmanagement und die Sozialwirtschaft ausbilden, kamen so viele Beiträge zusammen, dass sie nicht mehr in einen Band aufgenommen werden konnten. Es liegen jetzt drei Bände zum Thema vor (Wöhrle 2012a).

Es ist also an der Zeit, erstens eine subjektive Einschätzung abzugeben, auf welchem Stand sich die Diskussion über das Sozialmanagement - als »Management in der Sozialwirtschaft « - befindet. Zweitens sollen daraus Empfehlungen formuliert werden, die dem Manager 
und der Managerin in der Praxis weiterhelfen können.

\section{Die sieben Managementmodelle der Wirtschaft}

Beim Versuch, den gegenwärtigen Diskussionsstand zu markieren, wird deutlich, dass er sehr den Diskussionen in der Managementliteratur für die Wirtschaftsunternehmen in den 1990er Jahren ähnelt, als sich diese auf die Globalisierung einzustellen hatten (vgl. Wöhrle 2012b):

- Dort war von »Delfin-Strategien« (als Bild für den Sprung aus dem Gewässer) die Rede, um sich als Management eine Übersicht in unübersichtlicher Umwelt zu verschaffen,

- vom Umbau behäbiger Ozeanriesen (als Bild für große Organisationen, die in turbulenten Gewässern zu unbeweglich sind), zu wendigen Schnellbooten. Letztere, bestehend aus sich selbst steuernde Einheiten, sollten ei- genständig den Weg finden - wobei ein paar auch verloren gehen können. Hauptsache die Gesamtorganisation wird nicht beschädigt.

- Selbstverständlich wurde die Schwierigkeit des Umbaus entdeckt. Insbesondere die alten Vorstellungen von Organisationen als Maschinen mussten überwunden werden und es kamen systemtheoretische Modelle ins Blickfeld sowie Modelle, Organisationen als Kulturen zu betrachten. Als Anleitung für das Management fungierten Ansagen, einen Wandel zweiter Ordnung zu verfolgen, was nichts anderes bedeutet, als eine Revolution hinsichtlich alter Denkweisen und Routinen zu bewerkstelligen und sich nicht mit kosmetischen Korrekturen und Anpassungen zu begnügen.

- Und nun kamen die Berichte über gescheiterte Umbaumaßnahmen und die theoretischen Aufarbeitungen ins Blickfeld, mit denen festgestellt wurde, dass Patentrezepte für das Change Management meist nach dem Strick- muster verfasst wurden, dass ein zufällig für eine Organisation geglückter Umbauprozess nun vom Ende her zum Anfang "zurechtgeschrieben « wurde, um eine Anleitung für ein $\mathrm{Er}$ folgskonzept $\mathrm{zu}$ veröffentlichen und entsprechend $\mathrm{zu}$ beraten.

- Daneben wurden theoretische Grundlagen deutlich, mit denen eher erklärt werden kann, dass eine Organisation sich nicht ändern kann. Mit dem »Tunnelblick « (alle sehen die Anforderungen so, wie die Organisation es vorgibt, dass sie zu sehen sind), die Problemlösungsroutinen und die Anschlussfähigkeit der Entscheidungen (wodurch alles Neue ebenfalls nach dem Muster behandelt wird, nach dem die Organisation immer schon alles behandelt hat) können die Organisationen alles Neue nur auf das Maß klein arbeiten, mit dem sie schon immer umgehen konnten.

- Relativiert haben sich durch neuere Erkenntnisse in der Organisationssoziologie, Organisationspsychologie

\section{Managementkonzepte im Überblick}

Alle Managementkonzepte und Managementtechniken basieren auf der Führungstechnik und beziehen sich auf den »kooperativen Führungsstil«. Hier ist der Vorgesetzte in der Regel Koordinator und gibt Informationen in die Mitarbeitergruppe. Die Gruppe ist beim Entscheidungsfindungsprozess aktiv beteiligt. Es gibt folgende wesentliche Management-by-Konzepte:

- Management by Delegation, bei dem Aufgaben durch den Vorgesetzten an die Mitarbeiter übertragen werden, die diese vollständig hinsichtlich Entscheidungen und Abwicklung verantworten, z. B. Abwicklung eines vollständigen Kundenauftrages durch eine Mitarbeitergruppe. Eine deutsche Variante ist das Harzburger Modell.

- Management by Objectives, bei dem Vorgesetzter und Mitarbeiter gemeinsam Ziele vereinbaren. Der Mitarbeiter bestimmt selbständig den Weg der Zielerreichung. Abschließend findet ein Soll-Ist-Vergleich statt, zum Beispiel einer vereinbarten realistischen und exakten Zielvereinbarung.

- Management by Exception, bei dem Mitarbeiter in Normalsituationen und Routinefällen selbstständig entscheiden und handeln. Vorgesetzte greifen nur in Ausnahmefällen und Ausnahmesituationen ein. Zum Beispiel wird folgender Normalfall definiert: Gewährung von Rabatt bis zu einer Höhe von maximal 20 Prozent durch die Mitarbeiter.

- Management by Crisis, bei dem die Führung vom Management durch bewusste Herbeiführung oder Provokation von Krisen im Unternehmen erfolgt, um zu besseren Ergebnissen als bisher zu gelangen.

- Management by Results, bei dem die Führung durch Ergebnisüberwachung ausgeübt wird, indem die erzielten Ergebnisse vom Management einer laufenden und präzisen Kontrolle unterworfen werden.
- Management by Motivation, bei dem die Führung durch gezielte Motivation der Mitarbeiter erfolgt, indem den Unterstellten Anreize gegeben werden, die sie zu weiterer Leistungssteigerung anregen sollen.

- Das Management by Projects als Form der Teamarbeit, bei dem die Bewältigung bestimmter Projekte im Vordergrund steht, zum Beispiel die Errichtung eines neuen Werkes oder die Einführung eines effektiveren EDV-Systems.

Weitere Management-Konzepte sind:

- Das Lean Management als effektiveres und "schlankes Management", das zur Kosteneinsparung einerseits die hierarchischen Ebenen des Unternehmens zu verringern versucht und anderseits die Geschäftsprozesse nutzenbringender und kostengünstiger zu gestalten versucht.

- Das Business Process Reengineering-Konzept als Ausdruck des fundamentalen Überdenkens aller betrieblichen Prozesse, die hinsichtlich ihres Beginns, ihrer Elemente und ihres Endes genau zu definieren sind. Hinzu kommen Erkenntnisse des Customer-Relationship-Managements, die permanent in die Produktions- und Geschäftsprozessketten des Unternehmens optimierend einzuarbeiten sind.

- Das Integrierte Management als Qualitätsmanagement, als Umweltmanagement, als wertorientiertes Management, als Wissensmanagement und als System der Arbeitssicherheit.

- Das St. Galler Management-Modell als integriertes Modell zur Führung von Unternehmen, das die Gesamtheit aller Gestaltungs-, Lenkungs- und Entwicklungsprozesse umfasst, die das Unternehmensgeschehen bestimmen. Es ist ein vernetztes Informations-Entscheidungssystem.

Quelle: Internet http://de.wikipedia.org/wiki/Managementprozess 


\section{Glosse}

Eine Weisheit der Dakota-Indianer sagt: "Wenn Du entdeckst, dass Du ein totes Pferd reitest, steig ab! « Im modernen Management und in der Verwaltung haben sich zu dieser einfältigen Reaktion zahlreiche kreative Alternativen entwickelt:

1. Man besorgt eine stärkere Peitsche.

2. Man wechselt den Reiter aus.

3. Man gründet eine Projektgruppe, um zu analysieren, was mit dem toten Pferd los ist.

4. Man besucht anderer Orte und Organisationen, um zu sehen, wie man dort tote Pferde reitet.

5. Man erhöht die Qualitätsstandards für das Reiten toter Pferde.

6. Man startet ein Benchmarking, um verschiedene tote Pferde zu vergleichen.

7. Man erarbeitet differenzierte Kriterien, die festlegen, wann ein Pferd überhaupt tot ist.

8. Man schirrt mehrere tote Pferde zusammen in der Hoffnung, dann wieder reiten zu können.

9. Man richtet eine unabhängige Kostenstelle für tote Pferde ein.

10. Man strukturiert um, damit eine andere Abteilung das tote Pferd bekommt.

11. Man präsentiert PowerPoint-Folien, die zeigen, was das Pferd könnte, wenn es noch leben würde.

12. Man stellt fest, dass die anderen auch tote Pferde reiten, und erklärt dies zum Normalzustand.

und somit den Grundlagen der Managementlehre Vorstellungen eines Machertums im Management (also der lediglichen Durchstellbarkeit von Entscheidungen von oben nach unten). Für die Praxis entscheidend ist ebenfalls, dass die Mitarbeiter und Mitarbeiterinnen in ihrer Bedeutung für die Steuerung des Unternehmens im Sinne eines Intrepreneurship - verstanden als unternehmerisches Verhalten von Mitarbeitenden - deutlicher hervortreten.

- Auf diesem Hintergrund entsteht nun das Bild einer Lernenden Organisation, für deren Realisierung das Lernen der Individuen gebraucht, aber insbesondere das Lernen von Gruppen und die Weitergabe der Informationen in vernetzten Bezügen bis hin zur Komplexität des Lernens von Organisationen als systemtheoretisch verstandene Netzwerke oder als Kulturen begriffen werden muss.

\section{Auf der Suche nach eigenen Managementmodellen für die Sozialwirtschaft}

Die Diskussion über das Sozialmanagement und das Management in der Sozialwirtschaft hat (von wenigen Vorarbeiten abgesehen) in den 1990er Jahren begonnen. Dabei wurden kühn erste Konzepte vorgelegt, die allerdings bald relativiert wurden. Aus heutiger Sicht ist festzuhalten (vgl. Wöhrle 2012b):

- Die Vorreiter (wie Müller-Schöll, Maelicke, Schwarz) haben früh darauf aufmerksam gemacht, dass die Sozialwirtschaft als Wirtschaftssektor, dessen Bedeutung erst heute so langsam ins Blickfeld kommt, nicht länger als Anhängsel der öffentlichen Verwaltung betrachtet werden kann.

- Mit den sozialpolitischen Neuausrichtung unter der rot-grünen Koalition und mit der Einführung der wurden, kann heute ein gesicherter Bestand der Anforderungen aus fachlicher Sicht und der Bestimmung von Besonderheiten des Managens in der Sozialwirtschaft festgestellt werden.

- Hinsichtlich der konzeptionellen Antworten für Managementmodell und Managementkonzepte ist der Diskussionstand eher bescheiden. Er erreicht nicht das Niveau von Managementmodellen, wie sie beispielsweise mit dem St. Galler Managementmodell vorgelegt werden (Rüegg-Stürm 2003). Allerdings ist deutlich, dass versucht wird, die Komplexität zu verarbeiten.

Folgende Ergebnisse im Blick auf Sozialmanagementkonzepte und Konzepte des Managements in der Sozialwirtschaft lassen sich aus heutiger Sicht filtern (vgl. Wöhrle 2012a):

- Nachdem anfänglich jedwedes Konzept aus den Wirtschaftswissenschaften zu übertragen gesucht wurde, liegen heute differenzierte Untersuchungen hinsichtlich der Übertragung vor.

- Die schnelle Suche nach einem Managementkonzept wird nur noch in wenigen Monografien fortgesetzt. Sie ist durch eine Suche nach gesicherten Grundlagen, nach Kriterien für die Übertragung von Managementinstrumenten auf die Sozialwirtschaft, nach Ausbildungskonzepten und theoretischen Bezugspunkten in Kooperationszusammenhängen (z. B.

\section{"Durch die Suche nach einem eigenen}

\section{Managementkonzept sollte kein Aufruhr in die Organisation getragen werden"}

"Neuen Steuerung « in der öffentlichen Verwaltung wurden die eigenständigen Suchbewegungen nach einem Managementverständnis in den Organisationen der Sozialwirtschaft jäh unterbrochen und es wurde eine Ausrichtung auf ein wettbewerbsorientiertes, betriebswirtschaftliches Programm verlangt.

- Trotz extremer Abwehrkämpfe gegen das Sozialmanagement, die aus den Fakultäten der Erziehungswissenschaften und Sozialen Arbeit geführt der erwähnten INAS) und Verlagsreihen (z. B. bei Nomos oder beim Ziel-Verlag) ersetzt worden.

- In den Arbeiten verschiedener Autoren (wie Fröse, Grunwald, Merchel, Puch, Wöhrle) werden Ansätze für zusammenhängende Konzepte sichtbar, jedoch kann in keinem Fall bereits von einem ausgearbeiteten Sozialmanagementkonzept gesprochen werden (vgl. Wöhrle 2012b).

- Es gibt Forschungsbemühungen in der Schweiz, in denen bekannte Ma- 
nagementmodelle wie das St. Galler oder das Freiburger Modell systematisch daraufhin untersucht werden, ob sie den Anforderungen des Managements in der Sozialwirtschaft genügen (Bürgisser u. a., in: Wöhrle 2012a, Bd. 2).

- Es wird an eigenständigen Managementmodellen der Sozialwirtschaft gearbeitet (vgl. Social Impact Modell), mit dem der Zusammenhang zwischen der dem Aufgreifen sozialer Probleme, ihrer Bearbeitung und der Rechenschaftslegung unter Beachtung der Eigenheiten und besonderen Anforderungen an Organisationen der Sozialwirtschaft gearbeitet (vgl. Fritze/Maelicke/Uebelhart 2011; Wöhrle 2012a, Bd. 2).

- Und es wird von Betriebswirten (wie Schellberg) an der Verfeinerung von Instrumenten für die Sozialwirtschaft gearbeitet (vgl. Wöhrle 2012a, Bd. 2).

\section{Lehren für die Management-Praxis}

Was könnte nun dem Management in einer konkreten Organisation weiterhelfen, um ein eigenständiges Sozialmanagementkonzept oder ein Konzept für das Sozialmanagement zu entwickeln? Folgende Schritte sollten bei einer Organisationsentwicklung gegangen werden (vgl. Wöhrle 2005):

- Ziehen Sie eine externe Forschungsund Beratungseinheit zu Rate. Holen Sie sich niemanden, der Ihnen noch ein weiteres Managementkonzept oder Managementtool verkaufen will. Wenden Sie sich eher an eine Hochschule, die einen Sozialmanagement-Studiengang hat und Praxisforschung betreibt. Das ist zwar terminlich aufwendiger, aber es hat den Vorteil, dass es objektiv hinsichtlich der Forschung wird und wenig kostet.

- Suchen Sie auf jeden Fall nach Fachleuten, die etwas von der klassischen Methode der Organisationsentwicklung verstehen, denn es sollte kein Aufruhr in Ihre Organisation getragen werden, sondern die Beschäftigten sollten an den ablaufenden Prozessen Anteil haben und sie mitgestalten dürfen.

- Legen Sie alle Ihre Managementinstrumente und Managementtools offen auf den Prüfstand. Lassen Sie sie durch die externe Fachlichkeit und die eigenen Mitarbeiter und Mitarbeiterinnen bewerten.

- Stellen Sie die eruierten Auffälligkeiten und Problemstellungen der Fachdiskussion zur Verfügung. Lassen Sie weitere Fachkreise mit darüber nachdenken.

- Ziel sollte sein, ein übergreifenden Selbstverständnis für das eigene $\mathrm{Ma}$ nagement mit Selbstverpflichtung und ethisch begründeten Regeln aufzustellen, aus dem sich ein schlüssiges Konzept ableiten lässt, in das sich wiederum die verschiedenen Managementinstrumente und Managementtools begründet integrieren lassen. Wenn einzelne Steuerungselemente herausfallen oder quer stehen, sollte nach den Ursachen gesucht werden; möglicherweise müssen sie entfernt, ersetzt oder umgebaut werden.

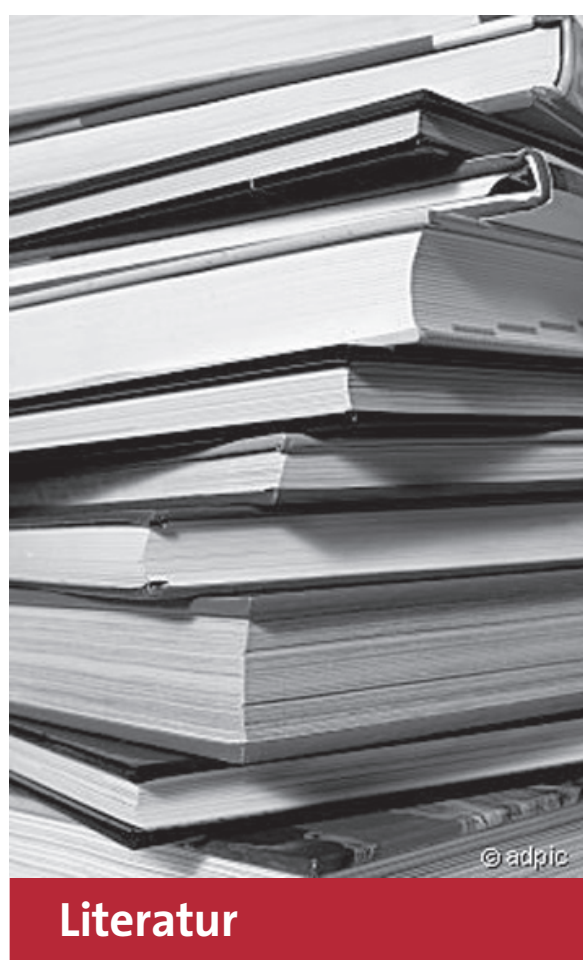

Bassarak, Herbert/Wöhrle, Armin: Sozialwirtschaft und Sozialmanagement im deutschsprachigen Raum. Bestandsaufnahme und Perspektiven, Augsburg (Ziel) 2008.

Boeßenecker, Karl-Heinz/Markert, Andreas: Studienführer Sozialmanagement. Studienangebote in Deutschland, Österreich und der Schweiz: Befunde-Analysen - Perspektiven, Baden-Baden (Nomos), 2. Auflage 2011. Deutsche Bank (2010): Deutsche Bank Research. Internet http://www.dbresearch. de/PROD/DBR_INTERNET_DE-PROD/ PRODOOOOOOO०00264932.ppdf.
- Wie bei jedem anderen Konzept auch, sollte es Reflexionsschlaufen geben, denn auch ein noch so schlüssiges Konzept sollte in gewissen Zeitabständen wieder auf den Prüfstand. Dies sollte zukünftig ein dem entstehenden Managementkonzept innewohnendes Element sein.

Am Ende des Prozesses sollte ein elegantes, leicht kommunizierbares und unmittelbar einsichtiges Managementkonzept stehen. Der Aufwand, dorthin zu gelangen, ist nicht gering. Die aus dem Ergebnis abgeleitete Führungs- und Steuerungspraxis sollte leicht handhabbare, schlanke Verfahren umfassen und von allen Organisationsmitgliedern mitgetragen werden.

Fritze, Agnés/Maelicke, Bernd/Uebelhart, Beat (Hg.): Management und Systementwicklung in der Sozialen Arbeit, Baden-Baden (Nomos) 2011. Grunwald, Klaus (Hg.): Vom Sozialmanagement zum Management des Sozialen? Eine Bestandsaufnahme, Hohengehren (Schneider) 2009.

Maelicke, Bernd (Hg.): Lexikon der Sozialwirtschaft, Baden-Baden (Nomos) 2008.

Puch, Hans-Joachim/Schellberg, Klaus: Sozialwirtschaft Bayern, Umfang und wirtschaftliche Bedeutung, Nürnberg 2010.

Rüegg-Stürm, Johannes: Das neue St.Galler Management-Modell: Grundkategorien einer integrierten Managementlehre: der HSG-Ansatz. Bern (Haupt) 2003.

Wöhrle, Armin: Den Wandel managen. Organisationen analysieren und entwickeln, BadenBaden (Nomos) 2005

Wöhrle, Armin: Zur Untersuchung des Sozialmanagements. Eine kritische Bestandsaufnahme und eine Vision, S. 139-178, in: Grunwald, K. (Hg.): Vom Sozialmanagement zum Management des Sozialen?, Hohengehren (SchneiderVerlag) 2009

Wöhrle, Armin (Hg.): Auf der Suche nach Sozialmanagementkonzepten und Managementkonzepten für und in der Sozialwirtschaft. Eine Bestandsaufnahme zum Stand der Diskussion und Forschung, Augsburg (Ziel) 2012a. Band 1: Übersicht, Einordnung und Bilanzen. Band 2: Verschiedene Blickwinkel und bisherige Managementkonzepte. Band 3: Entwürfe mit mittlerer Reichweite und Arbeiten an den Nahtstellen

Wöhrle, Armin: Sozialmanagementkonzepte und Managementkonzepte in der Sozialwirtschaft, Brandenburg (Service-Agentur des Hochschulverbundes Distance Learning) 2012 b. 